enabling the body size to increase.

But Tschanz's analysis, using different baselines, indicates that most of the growth of the neck of $T$. longobardicus during life can be explained as a strong and almost constant positive allometry: the neck length is linearly related to a power greater than unity of the animal's dimensions. Furthermore, a similar pattern of growth is found in the related but much smaller Macrocnemus bassanii. This needs confirmation from other species, but implies that the neck of T. longobardicus is as long as one would expect in any Tanystropheus of this size. Its great length in this species may therefore be an indirect consequence of the evolution of large size for some other reason, such as sexual competition or defence. Also, the positive allometry of neck growth in $T$. longobardicus seems to be weaker than in $M$. bassanii, hinting that this, the largest species of Tanystropheus, was approaching the longest viable length of neck - hardly surprising as the animal risked tipping over onto its nose every time it came out onto land. So, perhaps, $T$. longobardicus had a long neck simply because it was a big animal, and it survived in spite of it rather than because of it.

Michael A. Taylor is at the Leicestershire Museums, 96 New Walk, Leicester LE1 6TD, UK.

1. Bürgin, T., Rieppel, O., Sander, P.M. \& Tschanz, K. Scient Amer. 260 (6), 50-57 (1989)

2. Wild, R. Schweiz. paläont. Abh. 95, 1-162 (1973)

3. Wild, R. Mem. Soc. géol France N. S. 150, 37-44 (1987).

4. Tschanz, K. Palaeontology 31, 997-1011 (1988)

5. Massare, J.A. Paleobiology 14, 187-205 (1988)

6. Cox, C. B. Nature 254,654-655 (1975).

\title{
Controlling crystal growth
}

\section{Colin Humphreys}

EPITAXY, from the Greek 'arranged upon', describes a mode of crystal growth in which the atoms of one crystal are registered perfectly on the atoms of another. (DNA can also be regarded as a kind of epitaxial structure, Watson and Crick originally suggesting that one DNA helix 'crystallizes' on another which acted as a template.) Epitaxial structures are becoming increasingly important in materials science, physics, chemistry and electronics. For example, solid-state lasers can be built from epitaxially grown layers of two different semiconductors, say GaAs and AlGaAs. For most man-made structures the aim of epitaxy is to grow crystal A upon crystal B with an atomically flat and defect-free interface between A and B. But if the deposited material $A$ has a larger surface energy than the substrate B, A will roll up and form islands on B. Now, M. Copel et al. suggest (Phys. Rev. Lett. $\mathbf{6 3}, 632-635,1989$ ) a novel way to over come this problem: the use of a surfactant, which is a 'wetting agent' that changes the surface energy so that A spreads out as a thin film on B. The idea may eventually give increased control over epitaxial growth and enable epitaxial structures that have previously been thought impossible to be grown.

With real control of epitaxy, new materials could be fabricated atomic layer by atomic layer, each layer acting as a template for the next. This has yet to be achieved for alternating monatomic layers of different compounds, but the possibility that single crystals of such materials could have radically new properties makes the quest for perfect epitaxy a leading focus in materials science. The main impediment to this is that if $\mathrm{A}$ wets $\mathrm{B}$, then $\mathrm{B}$ does not wet $\mathrm{A}$, so that growing high quality $\mathrm{ABAB}$ multilayers is difficult and the use of surfactants is potentially very important.

In practice, epitaxy occurs according to one of three distinct growth modes as shown in the figure: layer-by-layer (Frank-van der Merwe (FM) growth); three-dimensional island growth directly on the substrate (Volmer-Weber (VW)); and a mixture of the above - initially layer-by-layer growth for a few layers followed by island growth (StranskiKrastanov (SK)). If A and B are identical materials then a single crystal can of course be grown, layer-by-layer, by various experimental techniques. But if $\mathrm{A}$ and $\mathrm{B}$ differ, their atomic spacings will differ and the result will be a strained epitaxial system. It can be shown theoretically that layer-by-layer growth of a strained system is not possible in equilibrium, which would seem to rule out the potential of epitaxial growth. Fortunately, kinetic constraints on mass transport can result in layer-by-layer growth and the resulting structures, although not in equilibrium, are metastable and can last for a long time.

As mentioned above, whether growth occurs layer-by-layer or via islands in such structures depends upon relative surface energies: if the deposited material $\mathrm{A}$ has larger surface energy than the substrate B, then island growth will tend to occur. (The situation is slightly more complicated than this because interface and strain energies must also be considered.) The problem is analogous to that of a liquid drop on a surface. Will it spread out to make a monolayer thin film (like oil on water) or will it form an island (like mercury on glass)?

Copel et al. have performed both

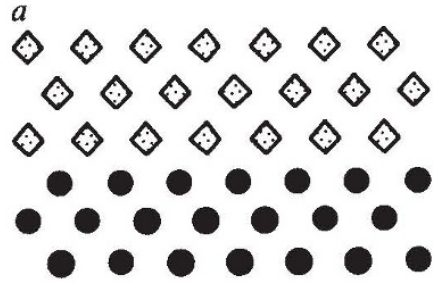

$b$
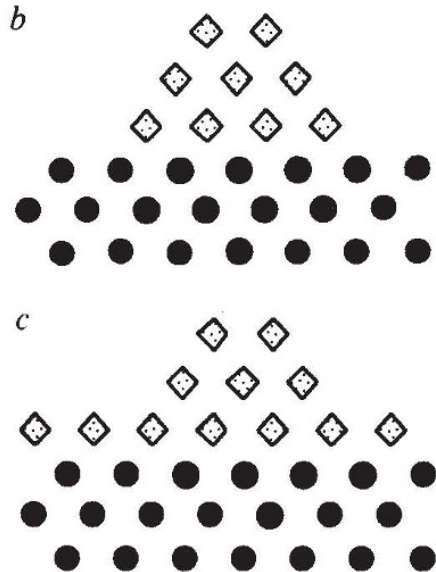

Three types of epitaxial growth: a, Frankvan der Merwe (FM); $b$, Volmer-Weber (VW); c, Stranski-Krastanov (SK).

calculations and experiments on the epitaxy of germanium and silicon, of which $\mathrm{Ge}$ has the lower surface energy. Hence, $\mathrm{Si}$ grows on $\mathrm{Ge}$ by island growth (VW mode), but Ge grows on Si initially layer-by-layer, then by island growth (SK mode). Copel et al. show that a monolayer of arsenic can be used to reduce the surface energies of both $\mathrm{Ge}$ and $\mathrm{Si}$. In addition, the As segregates to the surface during growth (there is little point in using a surfactant if it stays at the interface).

The authors claim that by covering the substrate surface with As, they promote the layer-by-layer growth of both $\mathrm{Ge}$ on $\mathrm{Si}$ and of $\mathrm{Si}$ on $\mathrm{Ge}$. If substantiated, this result will be very important. But further work is needed to characterize the quality of the epitaxial growth, and the extent to which As is incorporated in the growing layers must be more thoroughly investigated. Nevertheless, the control of epitaxial growth using surfactants as suggested by Copel et al. provides a potentially important new avenue to 'evade equilibrium thermodynamics' and produce high-quality epitaxial layers of structures that have previously proved impossible. We know now that enzymes mediate the growth of one strand of DNA upon another. Could biology be pointing the way to growth of perfect man-made epitaxial structures using mediators such as surfactants?

Colin Humphreys is in the Department of Materials Science and Engineering, Liverpool University, PO Box 147, Liverpool L69 3BX; from 1 January 1990 he will be in the Department of Materials Science and Metallurgy, Cambridge University, Cambridge CB2 3QZ, UK. 